\title{
On the Arf invariant in historical perspective
}

\author{
Falko Lorenz $\cdot$ Peter Roquette
}

Received: 13 January 2010 / Accepted: 12 February 2010 / Published online: 4 March 2010 (C) Springer-Verlag 2010

\section{Preface $^{1}$}

Originally this manuscript was prepared for my talk at the workshop on Sequences, Curves and Codes in Antalya, 25-29 September 2009. Later I had given a talk with the same title on October 5, 2009 at the conference on Positivity, Valuations, and Quadratic Forms in Konstanz.

In the discussion after the talk I learned that there may be an error in Arf's paper and perhaps his main theorem has to be modified. I am indebted to Karim Johannes Becher for this comment. Indeed, after another check I found the error in the proof of one of Arf's lemmas. Accordingly the manuscript had to be corrected, taking care of the situation and clarifying the scope of Arf's theorems after the correction.

For the convenience of the reader I have included in an appendix a counterexample and also a proof of the corrected version of Arf's theorem. This appendix has been prepared jointly with Falko Lorenz.

\section{Introduction}

In January 2009 I received a letter from the organizers of that workshop in Antalya with a friendly invitation to participate. The letter was accompanied by a bank note of 10 Turkish Lira. Reading the letter I found out that this was not meant as an advance honorarium for my talk, but it was to tell me that the note carried the portrait of

\footnotetext{
F. Lorenz

Münster, Germany

P. Roquette $(\square)$

Math. Inst., Univ. Heidelberg, 69126 Heidelberg, Germany

e-mail: roquette@uni-hd.de

${ }^{1}$ By Peter Roquette.
} 


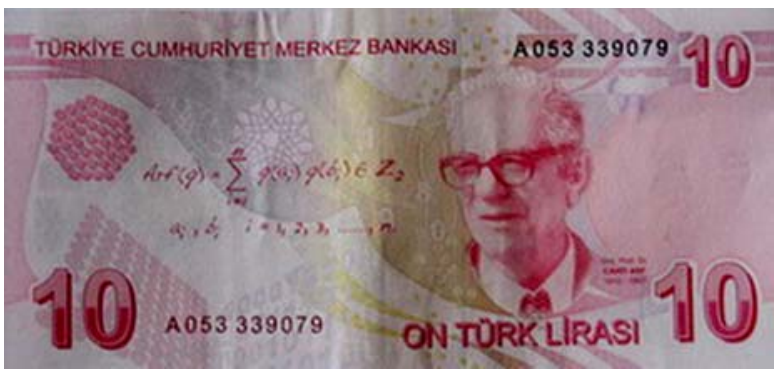

Fig. 1 Cahit Arf and its invariant

the Turkish mathematician Cahit Arf (1910-1997). Besides the portrait there appears some mathematical text pointing to Arf's discovery of what today is called the Arf invariant. Accordingly the organizers in their letter suggested that perhaps I would want to talk about the Arf invariant of quadratic forms.

It was my pleasure to follow this suggestion. Cahit Arf had been a Ph.D. student of Helmut Hasse in 1937/38. Arf's thesis [2] has become widely known, where he had obtained a generalization of a former theorem of Hasse about the ramification behavior of abelian number fields; today this is known as the "Hasse-Arf theorem". ${ }^{2}$ His next paper, after his thesis, contains the "Arf invariant" which is our concern today. This work too was inspired by a suggestion of Hasse. So the present report about the Arf invariant fits into my general project to investigate the mathematical contacts of Hasse with various other mathematicians, including Emil Artin, Emmy Noether, Richard Brauer and others, and now with Cahit Arf.

\section{Arf's first letter}

Much of what I know in this respect is based upon the letters between Hasse and his correspondence partners. Those letters are kept in the Handschriftenabteilung of the Göttingen University Library, they contain a rich source for those who are interested in the development of algebraic number theory in the 20th century. Among those documents there are preserved about 65 letters between Arf and Hasse from 1939 until 1975. ${ }^{3}$ We can see from them that in the course of time there developed a heartfelt friendship between the two.

The first ten letters are concerned with Arf's work on quadratic forms in characteristic 2. But where are the earlier letters, those about Arf's thesis? The answer is easy: There were no earlier letters, for during his graduate studies while composing his thesis, Arf worked at Göttingen University where Hasse was teaching. And people at the same university usually don't write letters but talk to each other.

\footnotetext{
${ }^{2}$ This generalization had been asked for by Artin in a letter to Hasse. For details from the historic perspective see, e. g., Sect. 6 of [22].

${ }^{3}$ In addition there are about 90 letters between Hasse and Arf's wife Halide, mostly in Turkish language, in which Hasse tried to practice and improve his mastery of the Turkish language.
} 
Fortunately for us, when Arf worked on quadratic forms in characteristic 2 he was back in Istanbul, and therefore the communication with his former academic teacher travelled by means of letters which are preserved. On October 12, 1939 Arf wrote to Hasse: ${ }^{4}$

Sehr geehrter Herr Professor,

Ich habe Ihren Brief vom 29.9.39 mit grosser Freude erhalten ... Ich habe jetzt eine unschöne Arbeit über quadratische Formen fast fertig geschrieben. Diese Arbeit wollte ich Ihnen vorlegen. Ich glaube aber, dass Sie jetzt wenig Zeit haben. Es handelt sich kurz um folgendes:

Sie hatten einmal den Wunsch geäußert, die Geschlechtsinvarianten einer quadratischen Form mit Hilfe der Algebrentheorie begründet zu sehen. Ich habe versucht dies zu tun. Da die Aufstellung dieser Invarianten für $p \neq 2$ fast trivial ist, habe ich gedacht, dass es nützlich sein würde wenn man zunächst die Theorie in Körpern von der Charakteristik 2 zu übertragen versucht. In der genannten Arbeit übertrage ich die Ergebnisse von Witt durch passende Änderungen in den Körpern von der Charakteristik 2 und ich gebe dann die vollständigen Invariantensysteme für arithmetische Äquivalenz der ternären und quaternären Formen in einem Potenzreihenkörper $k((t))$, wobei der Koeffizientenkörper $k$ die Charakteristik 2 hat und vollkommen ist ...

\section{Dear Professor,}

I am very glad to have received your letter of September 29, 1939 . . . I have almost completed the draft of a paper on quadratic forms. I had intended to submit it to you. But I believe that now you will not have much time for it. In short, the situation is as follows:

You had once expressed your wish to see the genus invariants of a quadratic form be established with the help of the theory of algebras. This I have tried to do. Since the compilation of those invariants is almost trivial in characteristic $\neq 2$ I thought it would be useful at first to try to transfer the theory to fields of characteristic 2. In the above mentioned paper I transfer the results of Witt by suitable modifications to fields of characteristic 2. And then I give a complete system of invariants for the arithmetic equivalence of ternary and quaternary forms in a power series field $k((t))^{5}$ where the field of coefficients $k$ is perfect of characteristic $2 \ldots$

From this we learn that it had been Hasse who had suggested the topic of Arf's investigation. Hasse's interest in quadratic forms stems from the time of his own thesis, 1923/24, when he had proved the Local-Global Principle for quadratic forms over number fields [10,11]. Later he had established the Local-Global Principle for central simple algebras over number fields, in cooperation with Emmy Noether and Richard Brauer [6]. There arose the question as to the mutual interrelation be-

\footnotetext{
${ }^{4}$ Observe the date of this letter. On September 1, 1939 World War II had started. Perhaps this was the reason why Arf believed that Hasse would not be able to devote much time to deal with Arf's paper since in war time Hasse may have been assigned other duties.

${ }^{5}$ I will use throughout my own notation and do not always follow the various notations in the original letters and papers.
} 
tween the theory of quadratic forms and the theory of algebras. Perhaps it would be possible to deduce the Local-Global Principle for quadratic forms from that for algebras?

This question (and more) had been answered beautifully in Witt's seminal paper [31]. At that time Witt held the position as assistant professor in Göttingen, and he was the leading member of the Arbeitsgemeinschaft in cooperation with Hasse. In his paper Witt associates to every quadratic form $f$ a central simple algebra $S(f)$ of 2-power index, called the Hasse algebra which, together with the dimension and the discriminant of the form, makes a complete set of invariants at least over global and local function fields.

Witt's paper represents a watershed in the theory of quadratic forms and it was the basis of the subsequent enormous expansion of the theory of quadratic forms. His biographer Ina Kersten says that this paper "ranks as one of his most famous works" [15]. However, Witt's theory covered only forms over a field of characteristic $\neq 2$. This is the point where Arf's paper comes in. He extended Witt's theory to fields of characteristic 2. In particular this applies to the case of local and global function fields of characteristic 2.

The desire to extend Witt's result to characteristic 2 had also been expressed by A. A. Albert in a paper which had just appeared in 1938 in the Annals of Mathematics [1]. There had been some letters exchanged between Albert and Hasse during the years 1931-1935 and we know that Albert's interest in the theory of quadratic forms over global fields had been encouraged by Hasse. ${ }^{6}$ The above mentioned paper by Albert shows that this interest continued. His paper is a kind of follow-up on Witt's [31] on quadratic forms: Albert first reproves, in his own way, some of Witt's results for global function fields and then shows that these hold also in the characteristic 2 case: namely that every quadratic form in 5 or more variables is isotropic. And then, in a footnote, he says about Witt's general theory:

The results of Witt on quadratic forms on a field of characteristic not two may probably be obtained for the characteristic two case only for forms with cross product terms. ${ }^{7}$ It would be very interesting to study the analogues of Witt's results for our characteristic two case but the author has not yet done so.

We see that Arf did just what was proposed here. I do not know whether Arf knew about Albert's paper and its footnote. In his own paper he cites Witt only and says in the introduction:

Die Anregung zu dieser Arbeit verdanke ich H. Hasse.

I owe to H. Hasse the suggestion for this work.

As we learn from Arf's letter, there was a second part in his manuscript where he investigates quadratic forms over rings of power series and their arithmetic invariants, at least for quadratic forms of low dimension. Again, the motivation for this comes from number theory. Due to his results in this second part Arf can be regarded

\footnotetext{
${ }^{6}$ See Chap. 8 in [23].

${ }^{7}$ It appears that Albert means quadratic forms which are "completely regular" in Arf's terminology. See Sect. 4.
} 
as a forerunner of the general theory of quadratic forms over rings, not necessarily fields. This is evident when we look at the book by Knus who gives a survey on quadratic forms over rings [16] where the notion of Arf invariant over rings is systematically treated. ${ }^{8}$

But in the end it turned out that Parts 1 and 2 were published separately. Part 1 appeared in Crelle's Journal where Hasse was editor [3]. Hasse would have liked to get also Part 2 for Crelle's Journal but it seems that there arose difficulties with the printing due to paper shortage in war times and so the second part appeared in the journal of the University of Istanbul. ${ }^{9}$

Here I will discuss only the first paper of Arf [3] where he introduces his Arf invariant. As already said in the preface, there is an error in Arf's paper which in turn reduces the scope of his main result. We shall discuss this in due course.

But before going into details let us familiarize a little with the people involved and with the time of the game.

\section{Some personal data}

Cahit Arf was born in 1910 in the town of Selanik which today is Thessaloniki. At that time it belonged to the Ottoman empire. But in the course of the Balkan war 1912 his home town was affected and the family escaped to Istanbul, later in 1919 to Ankara and finally moved to Izmir. ${ }^{10}$

Cahit Arf's childhood encompassed the Balkan wars, the World War I, the grand war at Gallipoli, the Greek invasion of western Anatolia and the invasion of Istanbul by the Allied Powers. When finally Turkey emerged as a new independent parliamentary republic in 1923 Cahit Arf was 13 years old. It was the beginning of a new era. The new Republic was hopeful, determined and full of invincible self confidence. These traits were also deeply trenched in young Arf. This would shape his attitude towards mathematics in the future.

In public school Arf's ease in mathematics was soon to be noticed by his teachers. In 1926 his father sent him to France to finish his secondary education at the prestigious St. Louis Lycée. Because of his extraordinary grades in mathematics he graduated in two years instead of the expected three years. Then he obtained a state scholarship to continue his studies at the École Normale Supérieur, again in France.

After his return to Turkey in 1932 Arf taught at high school and since 1933 he worked as instructor at Istanbul University.

It did not take him long to realize that he needed graduate study in mathematics. In 1937 he arrived at Göttingen University to study with Helmut Hasse.

\footnotetext{
${ }^{8}$ However we do not find Arf's Part 2 [4] cited in the bibliography of Knus' book.

${ }^{9}$ See [4]. The paper is usually cited for the year 1943 but at the end of the paper we read that it has been received on May 15, 1944.

10 The following citations are taken from the Arf biography written by Ali Sinan Sertöz [27]. There one can find more interesting information about the life, the work and the personality of Cahit Arf.
} 


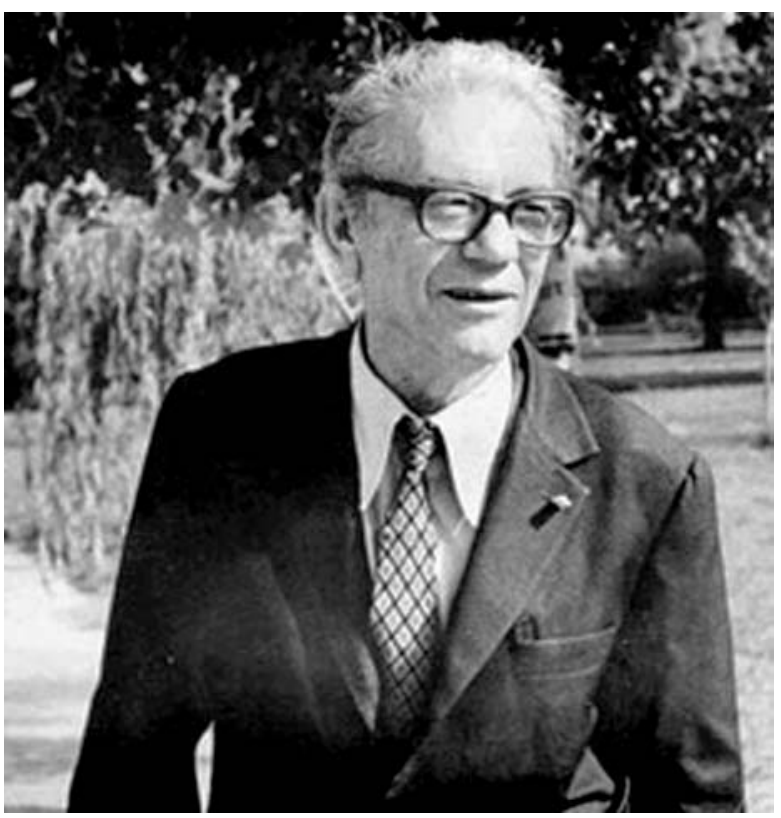

Fig. 2 Cahit Arf $1981^{11}$

At that time Arf was 27 years of age. I do not know why he had chosen Göttingen as his place of graduate study. Although Göttingen used to be an excellent mathematical center which was attractive to students throughout the world, that period had ended in 1933 when the new Nazi government decided to discharge the Jewish and the non-conformist professors; this had disastrous effects to the mathematical scene in Göttingen. It seems improbable that Arf had not heard about the political situation in Germany and its consequences for the academic life in Göttingen. Perhaps his mathematical interests at that time leaned towards algebra and arithmetics and he had found out (maybe someone had advised him) that in Göttingen there was Hasse who was known as an outstanding mathematician in those fields. In fact, measured by the high standard of Arf's thesis which he completed within one year after his appearance in Göttingen, it seems that already in Istanbul he had acquired a profound knowledge in the basics of modern algebra and algebraic number theory, and accordingly he may have chosen Göttingen because of Hasse's presence there.

Helmut Hasse was 38 years of age when in 1937 Arf arrived in Göttingen. Hasse was known as a leading figure in the development of algebraic number theory, in particular of class field theory. Just one year earlier in 1936 he had been chosen as an invited speaker at the International Congress of Mathematicians in Oslo. There he reported on his proof of the Riemann hypothesis for elliptic function fields over finite fields of constants; this proof had appeared in three parts in the 1936 issue of Crelle's Journal [12-14]. I would say that in those years Hasse was at the height of his math-

\footnotetext{
${ }^{11}$ Professor Ali Sinan Sertöz has kindly informed me that there is a photo showing Cahit Arf in Göttingen 1939, available at the address: http://www.mam.gov.tr/kutuphane/cahit_arf/index.html.
} 


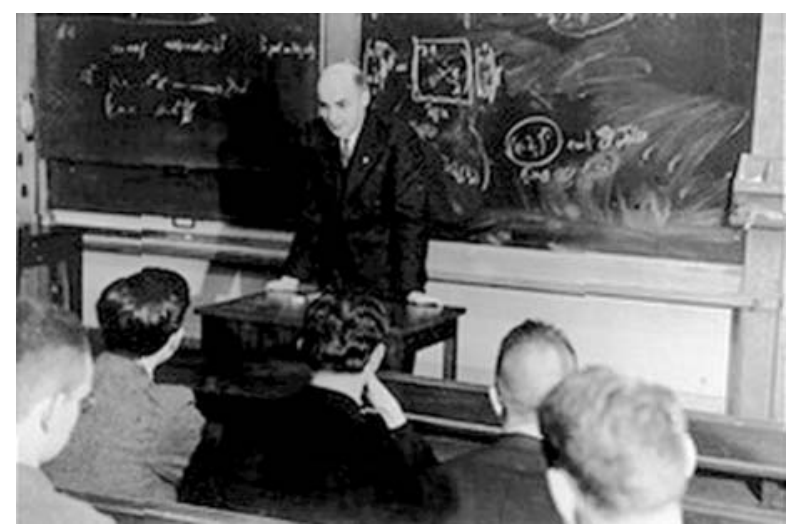

Fig. 3 Hasse lecturing in Göttingen

ematical power (notwithstanding a certain peak of his mathematical activities in the years after World War II). In 1934 Hasse had decided to leave the University of Marburg and to accept an offer to Göttingen. Hasse was not a Nazi but he described his political position as being patriotic. He strongly disagreed with the policy of expelling so many scientists from Germany; he considered this as a tragic loss of intellectual power in Germany. And he tried to do what he could to counteract this. When he decided to move from Marburg to Göttingen he did this with the expressed intention to restore, at least to a certain extent, the glory of Göttingen as an international place for mathematics. Although he spent a lot of time and energy on this, he could not be successful in the political situation. ${ }^{12}$

However, on a relatively small scale Hasse's activity in Göttingen had remarkable success. He managed to attract a number of highly motivated students to his seminar and the Arbeitsgemeinschaft. The latter was organized by Witt but Hasse participated at the meetings and led the mathematical direction of the work.

The high scientific level of the work in the Arbeitsgemeinschaft is documented in a number of publications in Crelle's Journal and other mathematical journals. Here we only mention volume 176 of Crelle's Journal which appeared just in 1937 when Arf came to Göttingen. A whole part of this volume ${ }^{13}$ contains papers which arose in the Arbeitsgemeinschaft and in the Seminar, of which Witt's famous paper on the socalled Witt vectors is to be regarded as a highlight. In the same volume (but in another part) appeared Witt's paper on quadratic forms [31] which, as said earlier already, has decisively influenced Arf's paper on his invariant [3].

Ernst Witt was 26 when he met Arf, hence one year younger. He had studied in Göttingen since 1930. He had received the topic of his Ph.D. thesis from Emmy Noether but since she had been dismissed she could not act as his thesis referee. ${ }^{14}$ The thesis was concerned with central simple algebras over function fields in the course

\footnotetext{
${ }^{12}$ For more facts from Hasse's biography see, e. g., Frei's biography [9], as well as Frei's recollections about Hasse in [8]. Hasse's involvement with the Nazi regime is discussed, e. g., in [25].

${ }^{13}$ Each volume of Crelle's Journal appeared in 4 parts (4 Hefte).

14 This was Herglotz with whom Witt maintained close relationship.
} 


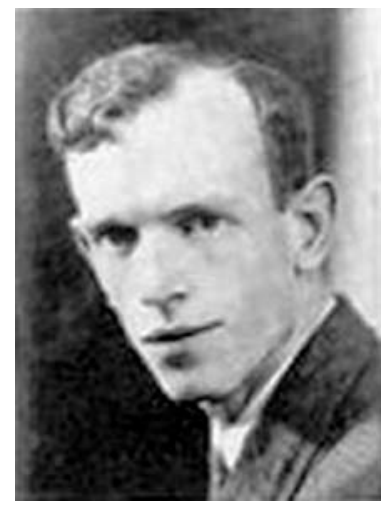

Fig. 4 Ernst Witt ${ }^{18}$

of which he proved the Riemann-Roch theorem for algebras, a ground breaking paper which nowadays attracts new interest in the setting of non-commutative algebraic geometry [30]. ${ }^{15}$ When Hasse came to Göttingen in 1934 he accepted Witt as his assistant on the recommendation of Emmy Noether. Witt has not many publications when compared to other mathematicians but every one is of high level and witnesses a profound insight into matematical structure. We have already mentioned his 1937 paper where he introduces "Witt vectors" [32]. ${ }^{16}$ Earlier the same year there had appeared his paper on quadratic forms [31] which actually constituted his Habilitation thesis. ${ }^{17}$

It seems fortunate that Arf in Göttingen had the chance to join the inspiring and motivated group of young mathematicians around Hasse, and among them Witt. There arose a friendship between the two which lasted for many years. It is without doubt that Arf's paper on quadratic forms in characteristic 2 has been influenced by Witt's in characteristic $\neq 2$.

\section{Quadratic spaces}

Let $K$ be a field.

Classically, a quadratic form over $K$ is given by an expression

$$
q(x)=\sum_{1 \leq i \leq j \leq n} a_{i j} x_{i} x_{j} \quad \text { with } \quad a_{i j} \in K .
$$

Two quadratic forms are said to be equivalent if one is obtained from the other by a non-degenerate $K$-linear transformation of the variables $x=\left(x_{1}, \ldots, x_{n}\right)$. An in-

\footnotetext{
${ }^{15}$ It is said that Witt completed the manuscript of his thesis within one week.

${ }^{16}$ It should not be forgotten that Witt vectors had been discovered somewhat earlier already by Hermann Ludwig Schmid [24] who also was in the Göttingen group around Hasse at that time. H. L. Schmid however worked with the main vector components only ("Hauptkomponenten") where the formulas for addition and multiplication are quite cumbersome. It was Witt who observed that the structural operations for Witt vectors can be described quite easily in terms of the ghost components ("Nebenkomponenten"). In this way he made the calculus of Witt vectors widely applicable.

${ }^{17}$ More biographic information about Witt can be obtained from Ina Kersten's biography [15] and the articles cited there.

${ }^{18}$ Source: [15] 
variant is a mathematical entity attached to quadratic forms which does not change if a form is replaced by an equivalent form.

Witt had replaced the above notion of quadratic form by the notion of quadratic space which was adapted to the "Modern Algebra" of the time. A quadratic space over $K$ is a vector space $V$ equipped with a function $q: V \rightarrow K$ and a bilinear function $\beta: V \times V \rightarrow K$ subject to the following conditions:

$$
\left.\begin{array}{rl}
q(\lambda x) & =\lambda^{2} q(x) \\
q(x+y) & =q(x)+q(y)+\beta(x, y)
\end{array}\right\} \quad \text { for } \lambda \in K, \quad x, y \in V .
$$

We assume $V$ to be of finite dimension $n$. If $u_{1}, \ldots, u_{n}$ is a basis of $V$ then any $x \in V$ may be written as $x=x_{1} u_{1}+\cdots x_{n} u_{n}$ with $x_{i} \in K$ and then $q(x)$ appears in the form Eq. 1 with $a_{i j}=\beta\left(u_{i}, u_{j}\right)$ for $i<j$ and $a_{i i}=q\left(u_{i}\right)$. In Witt's setup the notion of "invariant" now refers to isomorphisms of quadratic spaces instead of equivalences of quadratic forms.

It is common to interpret $q(x)$ as the "length" of the vector $x \in V$, more precisely as the square of its length. In fact, Witt and also Arf write $|x|^{2}$ instead of $q(x)$. Similarly $\beta(x, y)$ is interpreted as the "inner product" of the vectors $x$ and $y$ and accordingly Arf writes $x \cdot y$ for it. Witt however writes $x \cdot y$ for $\frac{1}{2} \beta(x, y)$ which is possible in characteristic $\neq 2$ and corresponds more to our geometric intuition, for then one has $x \cdot x=|x|^{2}$. In characteristic 2 however this is not possible and so we have $x \cdot x=2|x|^{2}=0$. In other words, in characteristic 2 we have to live with the fact that every vector is orthogonal to itself.

This has consequences. The first observation is that the process of diagonalization is not generally possible in characteristic 2 . Recall that in characteristic $\neq 2$ every quadratic form admits an equivalent "diagonal" form:

$$
q(x)=\sum_{1 \leq i \leq n} a_{i} x_{i}^{2} \quad \text { with } \quad a_{i} \in K .
$$

In Witt's terminology this means that every quadratic space $V$ admits an orthogonal basis $u_{1}, \ldots, u_{n}$, where $q\left(u_{i}\right)=a_{i}$. Thus $V$ splits as an orthogonal direct sum of onedimensional subspaces:

$$
V=\perp \sum_{1 \leq i \leq n}\left\langle u_{i}\right\rangle \quad \text { in char. } \neq 2 .
$$

But in characteristic 2 this is not always possible. Arf observed that one has to admit also two-dimensional subspaces:

$$
V=\perp \sum_{1 \leq i \leq r}\left\langle u_{i}, v_{i}\right\rangle+\perp \sum_{1 \leq j \leq s}\left\langle w_{j}\right\rangle \quad \text { in char. 2, }
$$

where $u_{i}$ and $v_{i}$ are not orthogonal to each other, i. e., $\beta\left(u_{i}, v_{i}\right) \neq 0$. After suitable normalization we may assume that

$$
\beta\left(u_{i}, v_{i}\right)=1^{19}
$$

\footnotetext{
${ }^{19}$ Arf however does not assume this and he admits for $\beta\left(u_{i}, v_{i}\right)$ any non-zero element in $K$. Therefore his formulas for the Arf invariant look a little more complicated than ours.
} 
The dimension $n$ of $V$ is $n=2 r+s$. Thus in characteristic 2 any quadratic form admits an equivalent form as follows:

$$
\begin{aligned}
q(x) & =\sum_{1 \leq i \leq r}\left(a_{i} x_{i}^{2}+x_{i} y_{i}+b_{i} y_{i}^{2}\right)+\sum_{1 \leq j \leq s} c_{j} z_{j}^{2} \\
\text { for } x & =\sum_{1 \leq i \leq r}\left(x_{i} u_{i}+y_{i} v_{i}\right)+\sum_{1 \leq j \leq s} z_{j} w_{j}
\end{aligned}
$$

where we have put

$$
a_{i}=q\left(u_{i}\right), \quad b_{i}=q\left(v_{i}\right), \quad c_{j}=q\left(w_{j}\right) .
$$

Arf speaks of "quasi-diagonalization" since only the second sum in Eq. 6 is in pure "diagonal form" whereas the matrix of the first sum splits into $2 \times 2$ submatrices along the diagonal. Note that in characteristic 2 the square operator is additive; accordingly the second sum in Eq. 6 is called the quasi-linear part of $q$. The second sum in Eq. 5 consists of all $z \in V$ which are orthogonal to $V$; therefore it is denoted by $V^{\perp}$.

A quadratic form is called regular if it has no equivalent form which can be written in fewer that $n$ variables. This is equivalent to saying that the coefficients $c_{j}$ appearing in the quasi-linear part of Eq. 6 should be linearly independent modulo the subfield $K^{2}$ of $K$. We also say that the corresponding quadratic space $V$ is regular; this means that $V^{\perp}$ does not contain any vector $z \neq 0$ with $q(z)=0$.

If $V^{\perp}=0$ then the quadratic space $V$ is called completely regular. ${ }^{20}$ We also say that the quadratic form is completely regular; this means that there is no quasi-linear part. The dimension of a completely regular space is even.

We see that in characteristic 2 we have two notions of regularity: Besides the ordinary notion there is the stronger notion of complete regularity. This is a phenomenon which does not appear in characteristic $\neq 2$. There, a quadratic form is regular if in the diagonal form Eq. 3 all coefficients $a_{i}$ are non-zero. ${ }^{21}$

\section{Clifford algebras}

The Clifford algebra $C(V)$ of a quadratic space $V$ is defined as an associative $K$ algebra (not necessarily commutative) generated by the $K$-module $V$ and with the defining relations:

$$
x^{2}=q(x) \quad \text { for } \quad x \in V
$$

In view of Eq. 2 this implies

$$
x y+y x=\beta(x, y) \quad \text { for } \quad x, y \in V .
$$

\footnotetext{
${ }^{20}$ In German "vollregulär".

${ }^{21}$ Warning: Sometimes in the literature, in characteristic 2 the terminology "regular" or "nonsingular" is used instead of Arf's "completely regular", while Arf's "regular" is called "semi-regular". This should be kept in mind in order to avoid misunderstandings.
} 
If $u_{1}, \ldots, u_{n}$ is a $K$-basis of $V$ then a $K$-basis of $C(V)$ is given by the products $u_{i_{1}} u_{i_{2}} \cdots u_{i_{k}}$ with $i_{1}<i_{2}<\cdots<i_{k}$ and $0 \leq k \leq n$. The $K$-dimension of $C(V)$ is $2^{n}$.

In view of its definition $C(V)$ is an invariant of $V$. So is the subalgebra $C_{0}(V) \subset$ $C(V)$ which is generated by the products $u_{i_{1}} u_{i_{2}} \cdots u_{i_{k}}$ with an even number $k$ of factors. The invariance of $C_{0}(V)$ is a consequence of the fact that the defining relations Eq. 7 are of degree 2. The dimension of $C_{0}(V)$ is $2^{n-1}$.

If $K$ is of characteristic 2 then we have the following rule:

$$
\text { If } V=V_{1} \perp V_{2} \text { then } C(V)=C\left(V_{1}\right) \otimes C\left(V_{2}\right) \text {. }
$$

Here I have written $V_{1} \perp V_{2}$ to indicate the orthogonal direct sum of $V_{1}$ and $V_{2}$. The tensor product is taken over $K$ as the base field. The validity of Eq. 9 is immediate if we observe that any $x \in V_{1}$ and $y \in V_{2}$ are orthogonal to each other, i. e., $\beta(x, y)=0$. Hence from Eq. 8 we conclude that $x y=-y x=y x$ showing that $C\left(V_{1}\right)$ and $C\left(V_{2}\right)$ are elementwise commutable. At the same time we see that in characteristic $\neq 2$ this is not the case since the appearing minus sign cannot be disregarded.

In view of the decomposition Eq. 4 we obtain for characteristic 2 that the Clifford algebra $C(V)$ decomposes into the tensor product of $r$ factors $C\left(\left\langle u_{i}, v_{i}\right\rangle\right)$ of dimension 4 , and the factor $C\left(V^{\perp}\right)$ of dimension $2^{s}$. The latter is the center of $C(V)$ and does not appear if $V$ is completely regular.

\section{Binary quadratic spaces}

First Arf investigates the Clifford algebra of a binary space $V=\langle u, v\rangle$ which is assumed to be completely regular:

$$
q(u)=a, \quad q(v)=b, \quad \beta(u, v)=1
$$

The Clifford algebra $C(V)$ is given by the defining relations

$$
u^{2}=a, \quad v^{2}=b, \quad u v+v u=1 .
$$

This is a central simple algebra of dimension 4 over $K$, i. e., a quaternion algebra, with the $K$-basis $1, u, v, u v$.

The even subalgebra $C_{0}(V)$ is of dimension 2 and has the basis elements 1 and $u v$ with the relation:

$$
(u v)^{2}+u v=u v(v u+1)+u v=u^{2} v^{2}=a b .
$$

Putting $w=u v$ and introducing the Artin-Schreier operator $\wp(X)=X^{2}+X$ we may write this as:

$$
C_{0}(V)=K(w) \quad \text { with } \quad \wp(w)=a b .
$$

For simplicity, suppose first that $a b \notin \wp(K)$. Then $K(w)$ is a separable quadratic field extension. According to the Artin-Schreier theory this field is uniquely determined 
by the residue class of $a b$ modulo $\wp(K)$. The nontrivial automorphism of $K(w)$ is given by conjugation with $u$ :

$$
u^{-1} w u=v u=w+1 .
$$

Since $u^{2}=a$ we see that $C(V)$ is a cyclic crossed product of the separable quadratic field $K(w)$, whose factor system is determined by the element $a \in K^{\times}$modulo the norm group from $K(w)^{\times}$.

The original quadratic form $q(x, y)$ can be rediscovered (up to equivalence) from these data by the norm function $N: K(w) \rightarrow K$ as follows:

$$
\begin{aligned}
a \cdot N(x+y w) & =a \cdot(x+y w)(x+y(w+1)) \\
& =a x^{2}+x(a y)+b(a y)^{2} \\
& =q(x, a y)
\end{aligned}
$$

Although this is not the original quadratic form $q(x, y)$, it is equivalent to it.

The case $a b=\wp(c) \in \wp(K)$ is somewhat exceptional since in this case $K(w)$ is not a field but a commutative separable $K$-algebra which decomposes into the direct product of two copies of $K$. Putting $e_{1}=w+c$ and $e_{2}=e_{1}+1$, these are idempotents and $e_{1} e_{2}=0$, hence $K(w)=K e_{1} \oplus K e_{2}$. By suitable choice of the basis $u, v$ of $V$ one can achieve that $u^{2}=a \neq 0$; then $u$ admits an inverse in $A$ and conjugation with $u$ induces an automorphism of $K(w)$ which permutes $e_{1}$ and $e_{2}$. Hence again ${ }^{22}, C(V)$ is a crossed product of $K(w)$, determined by the element $a \in K$ modulo norms from $K(w)^{\times}$. But every $a \in K$ is a norm from $K(w)$ in this case, and therefore $C(V)$ splits, i. e., it is a full matrix algebra over $K$. The formula Eq. 14 is still valid. In this way the case $a b \in \wp(K)$ appears quite analogous to the case $a b \notin \wp(K)$.

But there is one essential difference. If $a b \notin \wp(K)$ then the quadratic form $q(x, y)$ is anisotropic, i. e., $q(x, y)=0$ only for $x=y=0$. This we see from Eq. 14 since the norm function $N(z) \neq 0$ if $z \neq 0$. But if $a b \in \wp(K)$ then $q(x, y)$ is isotropic since $N\left(e_{1}\right)=N\left(e_{2}\right)=0$. In this case it turns out that the quadratic form is equivalent to $x y$. The corresponding quadratic space $V$ is called the hyperbolic plane and denoted by $H$. In any case, the residue class of $a b$ modulo $\wp(K)$ is an invariant of $V$ since it is determined by $K(w)=C_{0}(V)$. Arf defines: ${ }^{23}$

$$
\operatorname{Arf}(V): \equiv a b \bmod \wp(K)
$$

This is the Arf invariant of $V$ in the binary case. And the above discussion leads to the following

\footnotetext{
${ }^{22} K(w)$ is a commutative separable algebra over $K$ with an automorphism group $G$ of order 2 (the group interchanging the two copies $K e_{1}$ and $K e_{2}$ of $K$ ). Thus $K(w)$ is a quadratic "Galois algebra". The theory of crossed products of Galois algebras can be developed in complete analogy to the theory of crossed products for Galois field extensions. The first who had done this explicitely seems to be Teichmüller in his paper [29]. His terminology was "Normalring" for what today is called "Galois algebra". - Quite generally, for cyclic algebras we refer the reader to [23] and to [20].

${ }^{23}$ Arf in his paper writes $\Delta(V)$.
} 
Theorem: Let $V=\langle u, v\rangle$ be a two-dimensional completely regular quadratic space, so that Eq. 10 holds. The Clifford algebra $C(V)$ together with the Arf invariant $\operatorname{Arf}(V)$ completely determine $V$ up to isomorphism. $V$ is isotropic if and only if $\operatorname{Arf}(V) \equiv 0 \bmod \wp(K)$, and then $V$ is a hyperbolic plane.

Remark 1 Central simple algebras in every characteristic $p>0$ with defining relations

$$
u^{p}=a, \quad \wp(w)=c, \quad u w=(w+1) u
$$

had been systematically studied earlier, in particular by Teichmüller in his paper [28]. Such an algebra is called p-algebra. Teichmüller denotes it by $(a, c] .{ }^{24}$ In the case $p=2$ we obtain a quaternion algebra $A$. In view of the above considerations we see that $A=(a, c]$ is the Clifford algebra $C(V)$ of the binary quadratic space $V=\langle u, v\rangle$ with Eq. 10 for $b=a^{-1} c$. Whereas the relations Eq. 11 represent the description of $A$ as the Clifford algebra of the given quadratic space $V$, the relations Eq. 16 put into evidence the description of $A$ as a crossed product of some separable quadratic extension $K(w)$ of $K$ with $\wp(w)=c$. The connection between the two is given by the fact that $c$ is the Arf invariant of the space $V$, while the quadratic form of $V$ is given by the norm form of $K(w)$ multiplied by $a$; see Eq. 14.

The paper of Teichmüller mentioned above appeared in 1936, one year before Arf came to Göttingen. In this paper Teichmüller studies, among other things, the conditions for two such $p$-algebras $(a, c]$ and $\left(a, c^{\prime}\right]$ to be isomorphic. If $p=2$ then this result has some bearing on Arf's investigations. It would have been desirable that Arf cites Teichmüller's paper and points out the connection between his and Teichmüller's investigation. However Arf did not do this. Why not? Did he not know Teichmüller's paper?

Teichmüller had been a very active member of the Göttingen Arbeitsgemeinschaft but he had left for Berlin in early 1937. Hence Arf had probably not met Teichmüller. But certainly Teichmüller's results were known and valued in Göttingen and Arf must have heard about it. I find an explanation for Arf's silence about Teichmüller's work in a certain character trait of Arf which is mentioned in the biography of Sertöz [27]:25

Arf was in the habit of encouraging young mathematicians to discover mathematics by themselves rather than to learn it from others. To support his cause he would tell how in his university years, i. e., his École Normale years in Paris, he would never attend classes ... but proceed to develop that theory himself.

It seems that during his stay in Göttingen Arf had proceeded similarly, for Sertöz reports in his biography:

\footnotetext{
${ }^{24}$ Apparently this notation had been chosen to signalize the fact that the symbol $(a, c]$ is not symmetric in $a$ and $c$. Compare it with the notation $(a, b)$ for a quaternion algebra in characteristic $\neq 2$, given by the defining relations Eq. 18 below. That symbol is symmetric in the sense that $(a, b)=(b, a)$.

25 The story has been confirmed to me by several Turkish colleagues who had known Cahit Arf personally.
} 
Years later in Silivri, Turkey, Hasse would recall that after taking his problem ${ }^{26}$ Arf had disappeared from the scene for a few months only to come back with the solution.

This suggests to me that when Arf in 1939 was back in Istanbul and worked on quadratic forms then again he had proceeded similarly, i. e., discovering the solution of his problem by himself and not consulting other people or papers. In fact, in his paper [3] Arf cites only one paper explicitly, namely Witt's on quadratic forms [31]. -

The above Theorem holds in characteristic 2 . Let us briefly compare it with the similar situation in Witt's paper for characteristic $\neq 2$ : In this case a binary quadratic space is of the form $V=\langle u, v\rangle$ with mutually orthogonal vectors $u$ and $v$ and instead of Eq. 10, Eq. 11 we have

$$
q(u)=a, \quad q(v)=b, \quad \beta(u, v)=0
$$

The Clifford algebra $C(V)$ is now given by the defining relations

$$
u^{2}=a, \quad v^{2}=b, \quad u v=-v u .
$$

Again, this is a quaternion algebra. In the theory of algebras it is often denoted by $(a, b)$. The even subalgebra $C_{0}(V)$ is of dimension 2 and has the basis $1, u v$ but this time with the relation:

$$
(u v)^{2}=-u v(v u)=-u^{2} v^{2}=-a b=d .
$$

where $d$ is the discriminant of $V$. Thus

$$
C_{0}(V)=K(\sqrt{d}) .
$$

If $d \notin K^{\times 2}$ then $^{27}$ this is a quadratic field extension whose non-trivial automorphism is given by transformation with $u$. And again, we conclude that $C(V)$ is a crossed product of $K(\sqrt{d})$ which splits if and only if $a$ is a norm from $L$.

If $d \in K^{\times 2}$ then $K(u v)$ is not a field but the direct product of two copies of $K$. In this case and only in this case the quadratic space is isotropic, and it turns out that in this case the corrsponding quadratic form is equivalent to $q(x, y)=x y$, the hyperbolic plane.

So we see that for binary quadratic spaces Arf's situation in characteristic 2 is quite similar to Witt's situation in characteristic $\neq 2$, the only difference being quite natural, namely that the quadratic splitting field of $C(V)$ is generated by $\sqrt{-a b}$ in the case of characteristic $\neq 2$, whereas in characteristic 2 it is generated by a root of the Artin-Schreier equation $\wp(x)=a b$. And we see already here in the binary case:

In characteristic 2 the Arf invariant $\operatorname{Arf}(V) \in K / \wp(K)$ is the analogue of the discriminant $d(V) \in K^{\times} / K^{\times 2}$ in characteristic $\neq 2$.

This was the guiding idea of Arf when he wrote his paper.

\footnotetext{
${ }^{26}$ Namely the problem for his Ph.D. thesis.

${ }^{27} K^{\times}$denotes the multiplicative group of the field $K$ and $K^{\times 2}$ is the group of squares.
} 


\section{Higher dimensional quadratic spaces}

Now let $V$ be an arbitrary completely regular quadratic space. From Eq. 5 we know that $V$ decomposes into an orthogonal direct sum of two-dimensional spaces:

$$
V=\sum_{1 \leq i \leq r} V_{i} \quad \text { where } \quad V_{i}=\left\langle u_{i}, v_{i}\right\rangle
$$

and

$$
q\left(u_{i}\right)=a_{i}, \quad q\left(v_{i}\right)=b_{i}, \quad \beta\left(u_{i}, v_{i}\right)=1 \quad(1 \leq i \leq r)
$$

\section{Definition of Arf invariant:}

$$
\operatorname{Arf}(V) \equiv \sum_{1 \leq i \leq r} \operatorname{Arf}\left(V_{i}\right) \quad \bmod \wp(K)
$$

Recall that by definition $\operatorname{Arf}\left(V_{i}\right) \equiv a_{i} b_{i} \bmod \wp(K)$ so that this definition can also be written as:

$$
\operatorname{Arf}(V) \equiv \sum_{1 \leq i \leq r} q\left(u_{i}\right) q\left(v_{i}\right) \quad \bmod \wp(K) .
$$

This formula is printed on the 10-Lira note where, however, the underlying field is restricted to be $K=\mathbb{F}_{2}$, the prime field in characteristic 2 . In that case $\wp\left(\mathbb{F}_{2}\right)=0$ by Fermat's theorem and hence the congruence sign $\equiv$ in Eq. 22 can be replaced by equality.

If $r>1$ then it is not clear a priori that $\operatorname{Arf}(V)$ is an invariant of $V$. For, the definition Eq. 22 depends on how $V$ is decomposed into orthogonal subspaces $V_{i}$ in the form Eq. 19. One has to show that for every two such decompositions the corresponding sums in Eq. 21 are in the same class modulo $\wp(K)$. Arf does it in his paper but the proof requires some cumbersome computations. In later years Witt [33] and Klingenberg [17] have given simplified descriptions of $\operatorname{Arf}(V)$ from which one can see more directly its invariance. In the comments to Witt's paper in [34] the editor Ina Kersten reports:

It was Witt's concern in the fifties to eliminate the assumption that the characteristic of the ground field is different from 2.

Thus we see that Witt had carefully read Arf's paper and tried not only to simplify Arf's proof but also to extend it in such a way that it leads to a unified theory of quadratic forms, independent of the characteristic. In particular Kersten mentions Witt's cancellation theorem (see Sect. 8 below) and his attempts to investigate in detail the geometric situation which guarantees its validity.

Today we would verify the invariance of $\operatorname{Arf}(V)$ by investigating in more detail the structure of the Clifford algebra $C(V)$. We have already said in Sect. 5 that $C(V)$ contains a subalgebra $C_{0}(V)$ which is canonically defined by $V$, namely: $C_{0}(V)$ is generated by the products with an even number $k$ of factors in $V$. And in Sect. 6 we have seen that in the binary case, $C_{0}\left(V_{i}\right)=K\left(w_{i}\right)$ is a quadratic extension defined by the relation $w_{i}^{2}+w_{i}=a_{i} b_{i}$ which shows, using Artin-Schreier theory, that the class 
$\operatorname{Arf}\left(V_{i}\right)$ of $a_{i} b_{i}$ is an invariant of $C_{0}\left(V_{i}\right)$, hence of $V_{i}$. But Arf did not consider the subalgebra $C_{0}(V)$, probably he was not aware at that time that $C_{0}(V)$ was canonically defined by the quadratic space $V$. Therefore he had to use somewhat cumbersome explicit computations.

But using the invariance of $C_{0}(V)$, the following statement immediately shows that $\operatorname{Arf}(V)$ is an invariant: ${ }^{28}$

Proposition: Let $V$ be a completely regular quadratic space, represented as an orthogonal sum of two-dimensional spaces as in Eq. 19, Eq. 20. For each i let $C_{0}\left(V_{i}\right)=K\left(w_{i}\right)$ with $\wp\left(w_{i}\right)=a_{i} b_{i}$. Put $w=\sum_{i} w_{i}$, so that $\wp(w)=\sum_{i} a_{i} b_{i}$. Then the quadratic extension $K(w)$ equals the center of $C_{0}(V)$, and hence by Artin-Schreier theory the class of $\sum_{i} a_{i} b_{i}$ is an invariant of $V .{ }^{29}$

The essential part of the proof goes by verifying $w$ to commute with every element in $C_{0}(V)$. I recommend to verify this for $r=2$, i. e., two factors (and then using induction). One has to use that

$$
\begin{aligned}
C_{0}(V) & =C_{0}\left(V_{1}\right) \otimes C_{0}\left(V_{2}\right)+C_{1}\left(V_{1}\right) \otimes C_{1}\left(V_{2}\right) \\
& =K\left(w_{1}, w_{2}\right)+V_{1} \otimes V_{2}
\end{aligned}
$$

where $C_{1}\left(V_{i}\right)$ denotes the $K$-space generated by all products of an odd number of elements in $V_{i}$, hence $C_{1}\left(V_{i}\right)=V_{i}$. Show that $w=w_{1}+w_{2}$ commutes with $w_{1}$, with $w_{2}$ and with every product $x_{1} x_{2}$ with $x_{i} \in V_{i}$. (Use the fact that $w_{i} x_{i}=x_{i} w_{i}+x_{i}$.)

Let us mention that in Witt's situation of characteristic $\neq 2$ there arises a problem with the Clifford algebra $C(V)$. For, in general this is not a central simple algebra and it is not a product of quaternion algebras. For this reason in characteristic $\neq 2 \mathrm{Witt}$ replaced the Clifford algebra $C(V)$ by another algebra $S(V)$ which Witt has called "Hasse algebra"; this is defined as follows: First recall the notation $(a, b)$ for the quaternion algebra defined by the relations Eq. 18. Now consider the coefficients $a_{i}$ appearing in the diagonal form Eq. 3 and put $d_{i}=a_{1} a_{2} \cdots a_{i}$. Then the Hasse algebra is defined as the $n$-fold tensor product

$$
S(V)=\otimes \prod_{1 \leq i \leq n}\left(d_{i}, a_{i}\right) \sim \otimes \prod_{1 \leq i \leq j \leq n}\left(a_{i}, a_{j}\right)^{30}
$$

This is a central simple $K$-algebra and plays a role in Witt's theory of quadratic forms in characteristic $\neq 2$, analoguous to the Clifford algebra in characteristic 2 . But its definition Eq. 23 depends on the coefficients $a_{i}$ in the diagonal form Eq. 3. In order to show that it is an invariant, it is necessary to study the transformation from one diagonal form to an equivalent one. Witt's computations for this are similar to Arf's

\footnotetext{
${ }^{28}$ I have found this in the book by Knus [16].

${ }^{29}$ If $\wp(w) \equiv 0 \bmod \wp(K)$ then $K(w)$ is not a field but the direct sum of two fields isomorphic to $K$. We have discussed this situation already in the case of two-dimensional quadratic spaces.

${ }^{30}$ Quite generally we write $A \sim B$ if $A, B$ are central simple $K$-algebras which determine the same element in the Brauer group $\operatorname{Br}(K)$.
} 
computations for the invariance of $\operatorname{Arf}(V)$ in characteristic 2 . It seems to me that Arf had modelled his invariance proof for $\operatorname{Arf}(V)$ after Witt's invariance proof for $S(V) .{ }^{31}$

\section{Witt equivalence}

For any type of mathematical structures, the quest for invariants is motivated by the hope to be able to characterize the structures by their invariants (up to isomorphisms), and thus to obtain a classification of the structures under investigation. Here we are concerned with quadratic spaces $V$ in characteristic 2 and in particular with completely regular spaces. We now know three invariants:

1. the dimension $\operatorname{dim}(V)$,

2. The Clifford algebra $C(V)$,

3. the Arf invariant $\operatorname{Arf}(V)$.

For arbitrary fields we cannot expect that these three invariants characterize $V$ up to isomorphisms. But Arf wished to show that for special fields $K$ this is indeed possible. Although, as we shall explain, his proof of the main theorem cannot be upheld in its full generality, it turns out that the theorem is valid, e. g., over global and local fields $K$ in characteristic 2. In order to approach this problem, Arf follows Witt who had discovered the "Witt ring" by introducing a certain equivalence relation. As follows:

Recall that a quadratic space $V$ is called "isotropic" if there exists a vector $0 \neq x \in V$ with $q(x)=0$. The prototype of an isotropic completely regular space is the hyperbolic plane $H$ already introduced in Sect. 6. The corresponding quadratic form is $q(x, y)=x y$. The Arf invariant of $H$ is $\operatorname{Arf}(H) \equiv 0 \bmod \wp(K)$, and the Clifford algebra is $C(H) \sim 1$, which means that $C(H)$ splits. Arf proves the following

Theorem: (i) If the regular quadratic space $V$ is isotropic then $V=H \perp V^{\prime}$ where $V^{\prime}$ is uniquely determined by $V$ (up to isomorphisms).

(ii) Consequently, every regular quadratic space $V$ can be decomposed into an orthogonal sum of a number of spaces isomorphic to $H$ and a space $V^{*}$ which is anisotropic, and $V^{*}$ is uniquely determined by $V$ (up to isomorphisms).

The space $V^{*}$ is called the anisotropic kernel of $V$. Its quadratic form is called the kernel form ${ }^{32}$ of $V$.

As a consequence of this result Arf proves the general

Cancellation Theorem: Suppose the quadratic space $W$ is completely regular. If there exist regular quadratic spaces $V_{1}, V_{2}$ such that $W \perp V_{1} \cong W \perp V_{2}$ then $V_{1} \cong V_{2}$.

\footnotetext{
${ }^{31}$ We follow a suggestion of the referee and remark that the Hasse algebra $S(V)$ in characteristic $\neq 2$ is not an invariant of the class of $V$ in the Witt ring $\mathrm{WQ}(K)$ - contrary to the situation in characteristic 2 with the Clifford algebra (see the next section).

${ }^{32}$ In German: Grundform.
} 
In characteristic $\neq 2$ this famous cancellation theorem was contained in Witt's paper. Arf has observed that it holds also in characteristic 2, but only if $W$ is assumed to be completely regular. On the other hand, there is no such restriction necessary for $V_{1}, V_{2}$; they may be arbitrary regular quadratic spaces not necessary completely regular. Consequently the definition below of Witt equivalence applies to arbitrary regular spaces.

So Arf had obtained a new invariant of $V$, its kernel $V^{*}$. The original space $V$ is obtained from $V^{*}$ by adding an orthogonal sum of a number of hyperbolic planes, as many as the dimension of $V$ requires. We note that

$$
C(V) \sim C\left(V^{*}\right) \quad \text { and } \quad \operatorname{Arf}(V) \equiv \operatorname{Arf}\left(V^{*}\right) \bmod \wp(K)
$$

since $C(H) \sim 1$ and $\operatorname{Arf}(H) \equiv 0 \bmod \wp(K)$. We conclude:

In order to classify the quadratic spaces it is sufficient to classify the anisotropic spaces.

It is useful to work with the following

Definition of Witt equivalence: Two regular quadratic spaces (or quadratic forms) are Witt equivalent if they have isomorphic kernels. Notation: $V \sim W$.

This is indeed an equivalence relation for quadratic spaces. For completely regular spaces it blends with the orthogonal sum, i.e., if $V_{1} \sim W_{1}$ and $V_{2} \sim W_{2}$ then $V_{1} \perp V_{2} \sim W_{1} \perp W_{2} \cdot{ }^{33}$ The Witt classes of completely regular quadratic spaces with the operation $\perp$ form a group which we denote by $\mathrm{WQ}(K)$. We have $V \perp V \sim 0$, i. e., the elements of this group are of order 2 .

\section{Arf's Theorems}

Now we are able to state the main result of Arf's paper. For a special class of fields he wished to prove that the above invariants completely characterize the quadratic spaces.

Arf introduced the class of fields with the following property concerning the Brauer group. This property which we call (Q), refers to quaternion algebras:

(Q): The quaternion algebras over $K$ form a group within the Brauer group $\operatorname{Br}(K)$. In other words: If $A$ and $B$ are quaternion algebras then $A \otimes B \sim C$ where $C$ is a quaternion algebra again.

As I said earlier, Arf's main interest was directed to global and local fields of characteristic 2, i. e., power series fields and function fields over a finite field of constants, and also, more generally, over an arbitrary perfect constant field. Indeed, these fields were of particular interest to Hasse when he had suggested to Arf to work on quadratic forms in characteristic 2. It is not difficult to show that in characteristic 2

\footnotetext{
${ }^{33}$ If $V$ and $W$ are regular but not completely regular then $V \perp W$ need not be regular. Arf considers also this situation but then the relation $V \perp V \sim 0$ does not hold generally.
} 
these fields have the property (Q). But it seems that Hasse had not seen it immediately and so he asked Arf about it, who replied in a letter of March 29, 1940:

Wenn $A$ und $B$ normale einfache Algebren vom Grade 2 sind, so ist $A \otimes B$ höchstens vom Index 4. Da aber $\left[K^{\frac{1}{2}}: K\right]=2$ so enthalten $A$ und $B$ Teilkörper die zu $K^{\frac{1}{2}}$ isomorph sind. $A$ und $B$ enthalten also Elemente $u, v$ mit $u^{2}=v^{2} \in K$ die nicht zu $K$ gehören. Es gilt daher

$$
(u-v)^{2}=0 \quad \text { ohne, dass } \quad u-v=0 \quad \text { gilt. }
$$

$A \otimes B$ enthält also ein nilpotentes Element. Der Index von $A \otimes B$ ist daher höchstens 2 .

If $A$ and $B$ are central ${ }^{34}$ simple algebras of degree 2 then the index of $A \otimes B$ is at most 4 . But since $\left[K^{\frac{1}{2}}: K\right]=2$, both $A$ and $B$ contain subfields which are isomorphic to $K^{\frac{1}{2}}$. Hence $A$ and $B$ contain elements $u$ and $v$ respectively with $u^{2}=v^{2} \in K$, and $u$, $v$ do not belong to $K$. Hence we have

$$
(u-v)^{2}=0 \quad \text { but not } \quad u-v=0 .
$$

Thus $A \otimes B$ contains a nilpotent element. Therefore the index of $A \otimes B$ is at most 2 .

This settled Hasse's question but at the same time it showed that property (Q) holds for all fields with $\left[K^{\frac{1}{2}}: K\right]=2$.

Arf stated his main results in the form of two theorems.

Arf's Theorem 1: Assume that the field $K$ of characteristic 2 satisfies property (Q). Then any completely regular quadratic space $V$ of dimension $>4$ is isotropic. Consequently, its anisotropic kernel $V^{*}$ is of dimension $\leq 4 .{ }^{35}$

Arf's Theorem 2: Assume that the field $K$ of characteristic 2 has the property that every completely regular quadratic space of dimension $>4$ is isotropic. Then every completely regular quadratic space over $K$ is uniquely determined, up to isomorphism, by its Clifford algebra and its Arf invariant.

Certainly, Arf regarded his second theorem as the highlight of his paper. He had been able to accomplish his aim, namely to characterize quadratic forms by their invariants. His first theorem was to give a sufficient criterion for the field $K$ over which the classification could be done. However, as I have mentioned already, there is an error in the proof of Arf's first theorem and, in fact, there do exist counterexamples in the sense that his method of proof does not work under the hypothesis (Q). Hence Arf's Theorem 1 has to be corrected. It turns out that it holds for fields of imperfectness degree $\leq 1$. Recall that the degree of imperfectness $e=e(K)$ of a field $K$ of

\footnotetext{
${ }^{34}$ Arf used the terminology "normal" but nowadays it is usually said "central" to indicate that the center of the algebra equals the base field. - The $K$-dimension of a central simple $K$-algebra $A$ is a square $n^{2}$. The number $n$ is called the "degree" of $A$. The "index" of $A$ is defined to be the degree of the division algebra $D \sim A$.

${ }^{35}$ Arf also considered quadratic spaces $V$ which are regular but not completely regular. For those he claimed that any completely regular part of $V^{*}$ is of dimension $\leq 2$.
} 
characteristic $p>0$ is defined by

$$
\left[K: K^{p}\right]=p^{e} .
$$

$e=0$ means that $K$ is perfect. The condition $e=1$ appeared in the letter of Arf to Hasse of March 29, 1940 which we have cited above already. There, Arf pointed out that the condition $e=1$ implies condition (Q). Our correction of the theorem reads as follows:

Correction to Theorem 1. If $e(K)=1$ then every quadratic space over $K$ of dimension $>4$ is isotropic. If $e(K)=0$, i. e., if $K$ is perfect, then isotropy holds for every quadratic space of dimension $>2$.

For details of the corrected version I refer to the appendix. By the way, if the conclusion of Theorem 1 holds, i. e., if every quadratic space of dimension $>4$ is isotropic then condition (Q) is satisfied. (See the appendix.)

If $K$ is a function field of one variable over a perfect field of constants then $e(K)=1$. This classical result was also observed by Albert in his paper [1] which we have cited above already. On this basis Albert proved that quadratic forms of 5 variables over such function fields are isotropic. Thus in this sense one can state that Albert essentially had a proof of Arf's first theorem (in its corrected form) although he did not explicitely say so. Perhaps it is more correct to say that Arf had a proof of Albert's theorem since Albert had been first.

Apparently Arf did not know Albert's paper. When O.F.G. Schilling reviewed Arf's paper in the "Mathematical Reviews" he wrote: "The author is unaware of the work of A.A. Albert". We observe that Schilling did say this as a statement, not as a guess. Schilling had been a student of Emmy Noether in Göttingen and after Noether's emigration got his Ph.D. with Helmut Hasse in Marburg. Later he went to the USA. ${ }^{36}$ At the time when he wrote this review he held a position at the University of Chicago with Albert. He had kept contact to Hasse by mail, and on this occasions he had asked for information about the results in Hasse's Göttingen mathematical circle. It seems likely that he had been informed by Hasse or by someone else from Göttingen about Arf and his results; this enabled him to state that Arf "was not aware" of Albert's work and not adding "apparently" or something like this. Certainly Schilling himself knew Albert's papers.

Arf's (erroneous) proof of theorem 1 is not easy or straightforward but it is well arranged. It seems to me that Arf's style in his paper was much influenced by the suggestions and the advice of his academic teacher Hasse. For. in Arf's paper I have found a footnote which Hasse, being the editor of Crelle's Journal, had placed at the end of the introduction:

Anmerkung des Herausgebers: Im Einverständnis mit dem Verfasser habe ich dessen ursprüngliches Ms. überarbeitet.

Note by the editor: With the consent of the author I have revised his original manuscript.

\footnotetext{
${ }^{36} \mathrm{He}$ first stayed at the Institute for Advanced Study in Princeton where he had been accepted on the recommendation of Hasse who had written a letter to Hermann Weyl.
} 
We see that Hasse did with Arf's manuscript what he always did as an editor of Crelle's Journal, namely checking manuscripts carefully. As Rohrbach reports in [21]:

With his [Hasse's] characteristic conscientiousness, he meticulously read and checked the manuscripts word by word and formula by formula. Thus he very often was able to give all kinds of suggestions to the authors, concerning contents as well as form ...

So he did with Arf's paper. In the Hasse-Arf correspondence we read several times that Arf responds to changes suggested by Hasse, both approvingly and critically. Finally on February 8, 1941 Arf returned the final version to Hasse and wrote:

Mit gleicher Post schicke ich Ihnen die Korrekturbogen und das Manuskript der Arbeit über quadratische Formen zurück. Die Änderungen an drei Stellen die Sie vorgenommen haben scheinen mir unrichtig. Meine Gründe habe ich am Rand des Manuskripts geschrieben.

At the same time I am returning the galley proofs and the manuscript on quadratic forms. At three instances your proposed changes seem not to be correct. I have explained my reasons at the margin of the manuscript. ${ }^{37}$

The paper appeared in the same year 1941.

It seems curious that Hasse had not detected Arf's error although he was quite interested in the subject and had closely examined Arf's paper. This is even more curious since the error is of the same kind which many years ago, in 1927, Emmy Noether had committed in a similar situation and there resulted a close correspondence between Hasse and Emmy Noether about it. This correspondence finally led to their renowned theorem about cyclicity of algebras over number fields. It appears that in 1940 Hasse had forgotten that incident.

The situation back in 1927 had been as follows: ${ }^{38}$ Emmy Noether, in a letter to Richard Brauer of March 28, 1927, wrote to him that every minimal splitting field of a division algebra can be embedded into the algebra. Brauer knew that this was not the case and provided her with a counterexample. But this example seemed unnecessarily complicated to Emmy Noether; moreover she wished to know whether the counterexamples have bounded degree or not. So she wrote to Hasse, in a postcard of October 4, 1927 asking whether he could construct such counterexamples for quaternions. Hasse did so: He constructed cyclic fields of arbitrary high degree (over $\mathbb{Q}$ ) which were splitting fields of the classical quaternions but no proper subfield had this splitting property. Thus Noether as well as Hasse learned that one has to distinguish between minimal splitting fields and splitting fields of minimal degree; the latter indeed can be embedded into the algebra.

Now, in Arf's proof in the year 1940, a situation ocurred which was quite similar to the earlier one in 1927. On page 164, in the second paragraph of the proof of his "Satz 11", Arf considered two quaternion algebras $A_{1}, A_{2}$ (i. e., Clifford algebras of binary quadratic spaces $V_{1}, V_{2}$ ). He took quadratic extensions $K_{1}, K_{2}$ of

\footnotetext{
${ }^{37}$ I do not know which changes Hasse had proposed in those instances.

${ }^{38}$ I have told this story in detail in [23].
} 
$K$ which were splitting fields of $A_{1}$ and $A_{2}$ respectively, and he assumed $K_{1} \neq K_{2}$. Then $A_{1} \otimes A_{2}$ is split by the compositum $K_{1} K_{2}$ which has degree 4 over $K$, its Galois group being Klein's 4-group. In view of his hypothesis (Q) Arf knew that $A_{1} \otimes A_{2}$ is similar to some quaternion algebra, hence it admits a splitting field of degree 2 . And he argued that "necessarily" such splitting field can be found within $K_{1} K_{2}$. But this is not "necessarily" the case. In other words: $K_{1} K_{2}$ may be a minimal splitting field although it is not a splitting field of minimal degree.

Although Hasse's example of 1927 had referred to quaternions over $\mathbb{Q}$ we have here in characteristic 2 a similar situation. Why had Hasse not seen this error? We will never know. We know that in 1940 it was wartime and Hasse was drafted to the Navy. He worked at a Navy research institute in Berlin and could attend to his activities as an editor of Crelle's Journal in the evenings and on weekends only. So it seems that he did not check Arf's paper as thoroughly as he was used to in earlier times with other papers for Crelle's Journal.

\section{Perfect base fields}

If $K$ is perfect then, according to Arf's first theorem, every quadratic space $V$ of dimension $>2$ is isotropic. Consequently its anisotropic kernel $V^{*}$ is of dimension $\leq 2$. If $V$ and hence $V^{*}$ is completely regular and $V^{*}$ is not trivial then $\operatorname{dim} V^{*}=2$ and $C\left(V^{*}\right)$ is a quaternion algebra. But over a perfect field of characteristic 2 there exists only one quaternion algebra, namely the splitting one. Hence we conclude from Arf's second theorem that the Arf invariant alone is sufficient to characterize $V^{*}$. Thus the completely regular quadratic spaces over $K$ of given dimension correspond $1-1$ to the classes of $K / \wp(K)$. If $K$ is finite then $\wp(K)$ is an additive subgroup of index 2 in $K$ and hence there is essentially one anisotropic quadratic space. Its quadratic form is $q(x, y)=x^{2}+x y+b y^{2}$ where $b \notin \wp(K)$. If $K=\mathbb{F}_{2}$ then $b=1$.

In the literature Arf's Theorem is often reduced to this case. For instance, in the "Wikipedia" 39 we read the following:

In mathematics, the Arf invariant of a nonsingular quadratic form over the 2element field $\mathbb{F}_{2}$ is the element of $\mathbb{F}_{2}$ which occurs most often among the values of the form. Two nonsingular ${ }^{40}$ quadratic forms over $\mathbb{F}_{2}$ are isomorphic if and only if they have the same Arf invariant. The invariant was essentially known to Dickson (1901) and rediscovered by Cahit Arf (1941).

Certainly, this is all true. But does it provide an idea about the main discovery of Arf? In Arf's paper the field $\mathbb{F}_{2}$ is not mentioned at all, nor are finite fields. In a small remark, covering 4 lines only, Arf mentions how his theory applies in the case of perfect fields of characteristic 2. The main motivation and the main results of Arf are con-

\footnotetext{
${ }^{39}$ English version, August 20, 2009. A similar text, also restricted to the base field $\mathbb{F}_{2}$, appears in the "Encyclopedia of Mathematics".

${ }^{40}$ Here, "nonsingular" means "completely regular" in Arf's terminology. It seems that the author tacitly assumes that both these quadratic forms have the same number of variables.
} 
cerned with the description of quadratic forms by means of central simple algebras. This aspect is not even scratched in this article of Wikipedia.

The Wikipedia article seems to be written in view of the application of Arf's theory in topology. For, several of those applications are mentioned in the article. And indeed in topology one has to compute cohomology and other functors with coefficients modulo 2 which means that the base field is $\mathbb{F}_{2}$. An overview of the application of Arf invariants in topology is given by Turgut Önder in the appendix of the Collected Papers of Cahit Arf [5]. But this is not representative of Arf's work which is meant to exhibit the connection of quadratic forms in characteristic 2 with the theory of algebras.

By the way, in the 1901 book of Dickson on linear groups [7] which is mentioned in the Wikipedia article, also the case of an arbitrary finite base field of characteristic 2 is treated, not only $\mathbb{F}_{2}$. The fact that Arf did not cite this book may have one of two reasons: either he knew Dickson's book and found it is of no relevance for his investigation (which would be understandable), or he did not know it (which is more probable in view of his particular character trait which we have mentioned in Sect. 6). In any case, the statement that Arf has "rediscovered" what Dickson had known is misleading. Arf discussed a quite different theorem, and in a very special case this implies the statement of Dickson.

\section{Epilog}

After my conference talk I was asked about Arf's biography for the years after his paper on quadratic forms. I will not repeat here what is said in his biographies contained in $[5,27]$. Let me only mention that he became a prominent member of the Turkish scientific community (which is documented by the fact that his portrait decorates an official banknote) - but he also was a dedicated teacher. Many younger mathematicians in Turkey had been introduced by him into mathematics, he had encouraged them and showed them understandingly the way into our science. He is widely remembered in the mathematical community of Turkey. Robert Langlands, in his article about his impressions in Turkey, remembers warmly his discussions with Arf [19]. In particular Arf had directed Langlands' attention to a paper by Hasse on the local decomposition of the $\varepsilon$-factors; these factors appear in the functional equation of Artin's $L$-series. As Langlands says (English translation):

"I had rapid advance in my research having read Hasse's paper ... " and: “... thanks to Cahit bey, I solved this problem during my stay in Ankara and proved the existence of the local $\varepsilon$-factor."

I was also asked to report more extensively on the correspondence between Arf and Hasse, in particular the letters after 1941. I plan to do this some time in the future. Let me only mention that these letters, although they do not discuss anymore mathematics proper, show a growing friendship between the two. Hasse visited Turkey several times between 1957 and 1975. The last two preserved letters, dated March 1975 , concern the proposal to have an international colloquium on the structure of absolute Galois groups. This colloquium was planned by Arf jointly with M. Ikeda 
(who had earlier got a position in Turkey on the recommendation of Hasse). This conference took place in September 1975 in Silivri, a small village on the beach of the Marmara sea. I had the chance to participate in this conference and was able to observe the close friendly relationship between the two mathematicians, Arf and Hasse.

\section{Appendix: Proofs}

Arf's first theorem

$K$ denotes a field of characteristic 2. The degree of imperfectness $e=e(K)$ is defined by the formula

$$
\left[K: K^{2}\right]=2^{e} .
$$

If $e=0$ then $K$ is perfect and every quaternion algebra over $K$ splits. If $e=1$ then the quaternion algebras over $K$ form a subgroup of the Brauer group, as Arf had stated and proved in a letter to Hasse (see Sect. 9). If $K$ is a function field or a power series field with perfect field of constants then $e=1$.

Arf's theorem 1 (corrected). If $K$ is of imperfectness degree $e=1$ then every quadratic form of dimension $>4$ is isotropic. If $K$ is perfect then isotropy occurs for every quadratic form of dimension $>2$.

Today the so-called $u$-invariant $u=u(K)$ of a field $K$ is defined as the maximal dimension of anisotropic quadratic spaces over $K$. Thus Arf's first theorem says that $u \leq 2$ if $e=0$ and $u \leq 4$ if $e=1$. These are special cases of the following general

Proposition 1 Let $K$ be a field of characteristic 2. Then its $u$-invariant satisfies $u \leq$ $2^{e+1}$ where $e$ is the degree of imperfectness of $K$.

Proof Let $V$ be a quadratic space of dimension $n>2^{e+1}$. We are going to exhibit an element $0 \neq z \in V$ with $q(z)=0$. To this end we decompose $V$ in quasi-diagonal form as in Eq. 5:

$$
V=\sum_{1 \leq i \leq r}\left\langle u_{i}, v_{i}\right\rangle+\perp \sum_{1 \leq j \leq s}\left\langle w_{j}\right\rangle
$$

The number of subspaces appearing in this decomposition is $r+s$ while the dimension of $V$ is $n=2 r+s$. From each subspace we take an element $\neq 0$, say the $u_{i}(1 \leq i \leq r)$ and the $w_{j}(1 \leq j \leq s)$. The $r+s$ elements $q\left(u_{i}\right)$ and $q\left(w_{j}\right)$ in $K$ are linearly dependent over $K^{2}$. For, their number is

$$
r+s=n-r \geq n-\frac{n}{2}=\frac{n}{2}>\frac{2^{e+1}}{2}=2^{e}=\left[K: K^{2}\right] .
$$

(Observe that $r \leq \frac{n}{2}$.) Hence there is a nontrivial relation of the form

$$
\sum_{i} \lambda_{i}^{2} q\left(u_{i}\right)+\sum_{j} \mu_{j}^{2} q\left(w_{j}\right)=0 \quad \text { with } \quad \lambda_{i}, \mu_{j} \in K .
$$


Putting

$$
z=\sum_{i} \lambda_{i} u_{i}+\sum_{j} \mu_{j} w_{j} \in V
$$

we have $z \neq 0$ and $q(z)=0$.

Arf's second theorem

The theorem reads as follows:

Arf's theorem 2. Suppose that the field $K$ of characteristic 2 has the following property: Every completely regular quadratic space of dimension $>4$ is isotropic. Then every completely regular quadratic space $V$ is uniquely determined (up to isomorphism) by its dimension, the Brauer class of its Clifford algebra and its Arf invariant.

In particular the theorem holds for fields with $e(K)=0$ or 1 , according to Arf's theorem 1 in its corrected form. The following proof is essentially the same as in Arf's paper. We repeat it here for the convenience of the reader.

Proof The Arf invariant yields an additive homomorphism $V \mapsto \operatorname{Arf}(V)$ of the aditive group $\mathrm{WQ}(K)$ of Witt classes of completely regular spaces, into the $\wp$-factor group $K / \wp(K)$. The Clifford algebra yields an additive homomorphism $V \mapsto C(V)$ of $\mathrm{WQ}(K)$ into the Brauer group $\operatorname{Br}(K)$. First we claim:

If $V$ is completely regular then its Witt class is uniquely determined by the Brauer class of $C(V)$ and the $\wp$-class of $\operatorname{Arf}(V)$.

To this end we have to show: If $C(V) \sim 1$ in $\operatorname{Br}(K)$ and $\operatorname{Arf}(V) \equiv 0 \bmod \wp(K)$ then $V \sim 0$ in $\mathrm{WQ}(K)$. We may assume that $V$ is anisotropic. Then $\operatorname{dim} V \leq 4$ by hypothesis. Since $V$ is completely regular we have $\operatorname{dim} V=0,2$ or 4 . The case $\operatorname{dim} V=2$ is not possible since $\operatorname{Arf}(V) \equiv 0 \bmod \wp(K)$ implies $V=H$, the hyperbolic plane, hence $V$ would not be anisotropic. Suppose $\operatorname{dim} V=4$ and write $V=V_{1} \perp V_{2}$ as the orthogonal sum of binary spaces. Since $C(V) \sim 1$ we have $C\left(V_{1}\right) \sim C\left(V_{2}\right)$. Since both algebras have the same dimension it follows that they are isomorphic: $C\left(V_{1}\right)=C\left(V_{2}\right)$. Also, since $\operatorname{Arf}(V) \equiv 0 \bmod \wp(K)$ we have $\operatorname{Arf}\left(V_{1}\right) \equiv \operatorname{Arf}\left(V_{2}\right) \bmod$ $\wp(K)$. We have seen in Sect. 6 that a binary completely regular space is uniquely determined by its Clifford algebra and its Arf invariant. It follows $V_{1}=V_{2}$ hence $V=V_{1} \perp V_{1} \sim 0$, so again $V$ would not be anisotropic.

Now let $V$ be an arbitrary completely regular space, not necessarily anisotropic. Its Witt class contains a unique anisotropic space $V^{*}$. We have seen that $V^{*}$ is determined by the Arf invariant $\operatorname{Arf}(V)$ and the Brauer class of the Clifford algebra $C(V)$. In particular the dimension $n^{*}$ of $V^{*}$ is determined by those invariants. Now, if $\operatorname{dim} V=n$ then $V$ is obtained from $V^{*}$ by adding $\frac{n-n^{*}}{2}$ copies of the hyperbolic plane $H$. Hence $V$, and not only its Witt class, is determined by considering the dimension $n$ in addition to the invariants $\operatorname{Arf}(V)$ and $C(V)$. 


\section{A counterexample}

A counterexample to Arf's first theorem would be a field $K$ of characteristic 2 which admits an anisotropic quadratic space of dimension $>4$ and at the same time satisfies condition (Q). Now, such fields are difficult to construct. But the situation can be discussed on a more elementary level as follows.

The basic idea of the (erroneous) proof of Arf's first theorem had been as follows: Consider two binary completely regular quadratic spaces $V_{1}, V_{2}$ and the product $C\left(V_{1}\right) \otimes C\left(V_{2}\right)$ of their Clifford algebras. Using the fact that the product $C\left(V_{1}\right) \otimes$ $C\left(V_{2}\right)$ is similar to a quaternion algebra, Arf proceeds to show that the 5-dimensional quadratic space $V_{1} \perp V_{2} \perp K$ is isotropic. (Here, $K$ is regarded as a quadratic space by putting $q(z)=z^{2}$ for $z \in K$.) No other quaternion algebra appears in Arf's arguments, i. e., the condition (Q) is not fully used but only for the quaternion algebras $C\left(V_{1}\right)$ and $C\left(V_{2}\right)$. Therefore, the following yields a counterexample to his proof:

We shall construct a field $K$ of characteristic 2 and two completely regular binary quadratic spaces $V_{1}, V_{2}$ over $K$ with the following properties:

1. $V_{1} \perp V_{2} \perp K$ is anisotropic.

2. $C\left(V_{1}\right) \otimes C\left(V_{2}\right)$ is similar to a quaternion algebra. Equivalently, $C\left(V_{1}\right)$ and $C\left(V_{2}\right)$ admit a common quadratic splitting field.

The fact that a common quadratic splitting field implies the product to be similar to a quaternion algebra, is well known and finds its expression in the formulas

$$
\left(a_{1}, c\right]\left(a_{2}, c\right] \sim\left(a_{1} a_{2}, c\right] \quad \text { and } \quad\left(a, c_{1}\right]\left(a, c_{2}\right] \sim\left(a, c_{1}+c_{2}\right]
$$

for the crossed products. ${ }^{41}$ But the converse is also valid. For characteristic 2 this does not seem to be well known and therefore let us quickly present a proof:

Lemma 1 Let $A, B$ be quaternion algebras over $K$. Then $A \otimes B$ is similar to a quaternion algebra if and only if $A, B$ have a common quadratic splitting field.

Proof We assume that $A, B$ have no common quadratic splitting field and have to show that $A \otimes B$ is a division algebra. In particular our assumption implies that $A$ and $B$ are division algebras.

For any $a \in A$ let $K(a)$ denote the commutative subfield of $A$ generated by $a$. If $a \notin K$ then $K(a)$ is a splitting field of $A$. We have to show: If $K(a) \otimes B$ is a division algebra for every $a \in A$, and similarly $A \otimes K(b)$ is a division algebra for every $b \in B$ then $A \otimes B$ is a division algebra, i. e., every non-vanishing $z \in A \otimes B$ has an inverse.

Let $L=K(w)$ denote a separable quadratic subfield of $A$. There exists $u \in A$ whose inner automorphism induces in $L$ the nontrivial automorphism $\sigma$, i. e.,

$$
w^{\sigma}=u^{-1} w u .
$$

\footnotetext{
${ }^{41}$ The first formula concerns quaternion algebras with the common separable splitting field $K(w)$ with $\wp(w)=c$, and it is well known from Noether's general theory of crossed products. The second formula concerns quaternion algebras with the common inseparable splitting field $K(u)$ with $u^{2}=a$; it has been proved by Teichmüller [28] in the framework of his theory of $p$-algebras in characteristic $p>0$. Another proof is contained in Serre's book [26]. (Serre writes $[c, a)$ where we write $(a, c]$.)
} 
(Compare Eq. 13.) Every $z \in A$ has a unique representation in the form

$$
z=x+y u
$$

with $x, y \in K(w)$. If $z \in A \otimes B$ then again, $z$ has a unique representation of this form but now with $x, y \in K(w) \otimes B$. If $y=0$ then $z=x \in K(w) \otimes B$ has an inverse. So assume $y \neq 0$. After multiplying with $y^{-1}$ and changing notation we may assume that $z=x+u$ with $x \in K(w) \otimes B$.

We write $x=a+w b$ with $a, b \in B$. Then $x+u \in A \otimes K(a, b)$. If $a, b$ commute then $K(a, b)$ is a commutative subfield of $B$ and we conclude that $x+u$ has an inverse. So we may assume that $a, b$ do not commute.

We write $x^{u}=u^{-1} x u$ and compute

$$
(x+u)\left(x^{u}-u\right)=x x^{u}-u^{2} .
$$

(Note that $u x^{u}=x u$.) Here, $x x^{u} \in K(w) \otimes B$ and $u^{2} \in K$, and therefore $(x+u)$ $\left(x^{u}-u\right) \in K(w) \otimes B$. If $x x^{u}-u^{2} \neq 0$ we conclude that $(x+u)\left(x^{u}-u\right)$ has an inverse, and so does $x+u$. It remains to show that $x x^{u} \neq u^{2}$.

We have $x^{u}=a+w^{\sigma} b$ and compute

$$
\begin{aligned}
x x^{u} & =(a+w b)\left(a+w^{\sigma} b\right) \\
& =a^{2}+w w^{\sigma} b^{2}+w^{\sigma} a b+w b a \\
& =a^{2}+w w^{\sigma} b^{2}+\left(w+w^{\sigma}\right) a b+w(b a-a b) .
\end{aligned}
$$

Here, $w w^{\sigma}$ and $w+w^{\sigma}$ are in $K$, being the norm and the trace of the quadratic element $w$. Consequently the first three terms are contained in $B$. The last term is contained in $w B$ and it does not vanish since $a, b$ are supposed not to commute. Since $B$ and $w B$ are linearly disjoint over $K$ we conclude that $x x^{u} \notin B$ and in particular $x x^{u} \notin K$, hence $x x^{u} \neq u^{2}$.

Remark 21 . For fields of characteristic $\neq 2$ we have found this lemma in the book by Lam [18] who traces it back to Albert. Note that our proof works in any characteristic.

2. In characteristic 2 there are two kinds of quadratic splitting fields, separable and inseparable fields. Note that in the following construction, the common splitting fields are separable ones.

Our construction will be based on the following well known result.

Suppose that $K$ carries a discrete valuation with residue field $\bar{K}$ and prime element $t$. Consider a quadratic form $\bar{q}$ over $\bar{K}$ which we write as

$$
\bar{q}(x)=\sum_{1 \leq i \leq j \leq n} \bar{a}_{i j} x_{i} x_{j} \quad \text { with } \quad \bar{a}_{i j} \in K,
$$

not all coefficients vanishing. We lift each coefficient $\bar{a}_{i j}$ to an element $a_{i j} \in K$. We obtain a quadratic form over $K$, in the same number $n$ of variables:

$$
q(x)=\sum_{1 \leq i \leq j \leq n} a_{i j} x_{i} x_{j} \quad \text { with } \quad a_{i j} \in K .
$$


The $a_{i j}$ are in the valuation ring, not all in its maximal ideal. $q$ is called a "lift" of $\bar{q}$, and $\bar{q}$ is the "reduction" of $q$.

Lemma 2 In the situation explained above, if $\bar{K}$ admits an anisotropic quadratic form $\bar{q}$ of dimension $n$ then $K$ admits an anisotropic form of dimension $2 n$. In fact, if $q$ is a lift of $\bar{q}$ then the form $q+t q$ ( $t$ being a prime element) is anisotropic over $K$.

Proof If there would be a nontrivial relation $q(x)+t q(y)=0$ with the coordinates of $(x, y)=\left(x_{1}, \ldots, x_{n}, y_{1}, \ldots, y_{n}\right)$ in $K$ then, after multiplying $(x, y)$ with a suitable common factor we may assume that the $x_{i}, y_{j}$ are in the valuation ring and not all divisible by $t$. If there is one $\bar{x}_{i} \neq 0$ then reduction modulo $t$ gives a nontrivial relation $\bar{q}(\bar{x})=0$ in $\bar{K}$ which is impossible because $\bar{q}$ is anisotropic. Hence all the $x_{i}$ are divisible by $t$ whereas there is one $y_{j}$ not divisible by $t$. Writing $x=t x^{\prime}$ we obtain $t^{2} q\left(x^{\prime}\right)+t q(y)=0$. Dividing by $t$ and reducing modulo $t$ we obtain a nontrivial relation $\bar{q}(\bar{y})=0$, again a contradiction.

Construction of the counterexample: We start with a field $K_{0}$ and require that $K_{0}$ admits a separable quadratic extension field $L_{0}$. The norm form $L_{0} \rightarrow K_{0}$ is anisotropic. Let us denote it by $q$. Its Clifford algebra is the split crossed product of $L_{0} \mid K_{0}$; the usual notation ${ }^{42}$ is $\left(1, L_{0} \mid K_{0}\right)$ where 1 represents the factor system of the split crossed product of $L_{0} \mid K_{0}$.

Now let $K_{1}=K_{0}\left(t_{1}\right)$ be the rational function field in one variable $t_{1}$, equipped with the valuation belonging to the specialization $t_{1} \mapsto 0$, so that $t_{1}$ is a prime element. Let $L_{1}=L_{0}\left(t_{1}\right)$; this is an unramified separable quadratic extension of $K_{1}$. Its norm form $L_{1} \rightarrow K_{1}$ is a lift of the norm form $L_{0} \rightarrow K_{0}$, let us denote it also by $q$. According to the lemma the 4-dimensional form $q+t_{1} q$ is anisotropic over $K_{1}$. This is the orthogonal sum of the binary form $q$ and its scaled form $t_{1} q$. The corresponding Clifford algebras are $\left(1, L_{1} \mid K_{1}\right)$ and $\left(t_{1}, L_{1} \mid K_{1}\right)$. So they both admit the same quadratic splitting field $L_{1} \mid K_{1}$.

Now we repeat this process: Let $K_{2}=K_{1}\left(t_{2}\right)$ be a rational function field, equipped with the valuation belonging to the specialization $t_{2} \mapsto 0$. Let $L_{2}=L_{1}\left(t_{2}\right)$. This is an unramified separable quadratic extension of $K_{2}$. The norm form $L_{2} \rightarrow K_{2}$ is a lift of the norm form $L_{1} \rightarrow K_{1}$, let us again denote it by $q$. Again, the lemma shows that the 8-dimensional form $\left(q+t_{1} q\right)+t_{2}\left(q+t_{1} q\right)$ is anisotropic. It is the orthogonal sum of the four binary forms whose Clifford algebras are $\left(1, L_{2} \mid K_{2}\right),\left(t_{1}, L_{2} \mid K_{2}\right)$, $\left(t_{2}, L_{2} \mid K_{2}\right),\left(t_{1} t_{2}, L_{2} \mid K_{2}\right)$.

Thus all four have the same quadratic splitting field $L_{2} \mid K_{2}$.

Denote the corresponding binary quadratic spaces by $V, V_{1}, V_{2}, V_{12}$. Thus $V \perp$ $V_{1} \perp V_{2} \perp V_{12}$ is anisotropic. So is every quadratic subspace. Note that, by construction, $V$ belongs to a norm form and hence there exists $z \in V$ with $q(z)=1$. The one-dimensional space $K z$ is isomorphic to $K$ (as quadratic spaces). Now we obtain the subspace $K \perp V_{1} \perp V_{2}$ as the required counterexample.

Remarks 1 . The field $K_{0}$ may be chosen to be perfect. Then the rational function field $K_{2}=K_{0}\left(t_{1}, t_{2}\right)$ is of imperfectness degree $e\left(K_{2}\right)=2$. Our construction yields an anisotropic quadratic space of dimension 8 . Since the construction can be iterated we

42 in any characteristic 
obtain for every $e$ a field $K_{e}$ of imperfectness degree $e$ with an anisotropic quadratic space of dimension $2^{e+1}$. This shows that the bound given by Proposition 1 is best possible.

2. Instead of rational function fields one can use power series fields.

3. Our construction works in every characteristic. For characteristic $\neq 2$ we have found Lemma 3 in the book by Lam [18].

4. If every quadratic space of dimension $>4$ over $K$ is isotropic then the quaternion algebras over $K$ form a subgroup of the Brauer group.

Proof Let $A, B$ be two non-split quaternion algebras over $K$. We write $A=C(V)$, $B=C(W)$ where $V, W$ are binary completely regular quadratic spaces. We have $V \subset C(V)=A$. Similary $W \subset B$, hence $V+W+K \subset A \otimes B$. Here, $V+W+K$ is a 5-dimensional vector space which can be turned into a quadratic space by squaring within $A \otimes B$, i. e., $q(x)=x^{2}$ for $x \in V+W+K$. Since 5-dimensional quadratic spaces are isotropic by hypothesis, there exists $0 \neq x \in V+W+K$ with $x^{2}=0$, hence $A \otimes B$ is not a division algebra. This implies $A \otimes B$ is similar to some quaternion algebra, split or not.

By the way, a function field $K=K_{0}\left(t_{1}, t_{2}\right)$ in two variables over an algebraically closed field $K_{0}$ is a $C_{2}$-field, hence every quadratic space of dimension $>4$ is isotropic. It follows from Remark 4 that the quaternion algebras over $K_{0}\left(t_{1}, t_{2}\right)$ form a subgroup of the Brauer group. But we do not know whether the same is ever true for a field $K_{0}$ which is not algebraically closed.

\section{References}

1. Albert, A. A.: Quadratic null forms over a function field. Ann. Math. (2) 39, 494-505 (1938)

2. Arf, C.: Untersuchungen über reinverzweigte Erweiterungen diskret bewerteter perfekter Körper. J. Reine Angew. Math. 181, 1-44 (1939)

3. Arf, C.: Untersuchungen über quadratische Formen in Körpern der Charakteristik 2. I. J. Reine Angew. Math. 183, 148-167 (1941)

4. Arf, C.: Untersuchungen über quadratische Formen in Körpern der Charakteristik 2. II. Re. Fac. Sci. Univ. Istanbul (A) 8, 297-327 (1943)

5. Arf, C.: The Collected Papers. Turkish Mathematical Society (1990)

6. Brauer, R., Hasse, H., Noether, E.: Beweis eines Hauptsatzes in der Theorie der Algebren. J. Reine Angew. Math. 167, 399-404 (1932)

7. Dickson, L. E.: Linear groups: With an exposition of the Galois field theory. Dover, New York (1958). XVI, 312 S., reprint from the original edition 1901

8. Frei, G., Roquette, P. (eds.): Emil Artin and Helmut Hasse Their correspondence 1923-1934. With an introduction in English. Universitäts-Verlag, Göttingen (2008)

9. Frei, G.: Helmut Hasse (1898-1979). Expositiones Math. 3, 55-69 (1985)

10. Hasse, H.: Über die Äquivalenz quadratischer Formen im Körper der rationalen Zahlen. J. Reine Angew. Math. 152, 205-224 (1923)

11. Hasse, H.: Äquivalenz quadratischer Formen in einem beliebigen algebraischen Zahlkörper. J. Reine Angew. Math. 153, 158-162 (1924)

12. Hasse, H.: Zur Theorie der abstrakten elliptischen Funktionenkörper. I. die Struktur der Divisorenklassen endlicher Ordnung. J. Reine Angew. Math. 175, 55-62 (1936)

13. Hasse, H.: Zur Theorie der abstrakten elliptischen Funktionenkörper. II. Automorphismen und Meromorphismen. Das Additionstheorem. J. Reine Angew. Math. 175, 69-88 (1936)

14. Hasse, H.: Zur Theorie der abstrakten elliptischen Funktionenkörper. III. die Struktur des Meromorphismenrings. J. Reine Angew. Math. 175, 193-207 (1936) 
15. Kersten, I.: Biography of Ernst Witt. Contemp. Math. 272, 155-171 (2000)

16. M.-A. Knus: Quadratic and Hermitian Forms over Rings. Grundlehren der mathematischen Wissenschaften. Springer, Berlin Heidelberg New York (1980)

17. Klingenberg, W., Witt, E.: Über die Arfsche Invariante quadratischer Formen mod 2-. J. Reine Angew. Math. 193, 121-122 (1954)

18. Lam, T.Y.: Introduction to quadratic forms over fields, vol. 67 of Graduate Studies in Mathematics. American Mathematical Society, Providence, RI (2005)

19. Langlands, R.: Benim tanidigim Cahit Arf (Recollections of a year in Turkey with Cahit Arf) (turkish). Matematik Dünyasi (winter number) (2004)

20. Lorenz, F.: Algebra. Volume II: Fields with structure, algebras and advanced topics. Transl. from the German by Silvio Levy. With the collaboration of the translator. Universitext. Springer, New York (2008)

21. Rohrbach, H.: Helmut Hasse and Crelle's Journal. J. Reine Angew. Math. 500, 5-13 (1998)

22. Roquette, P.: On the history of Artin's $L$-functions and conductors. Seven letters from Artin to Hasse in the year 1930. Mitt. Math. Ges. Hamburg 19*, 5-50 (2000)

23. Roquette, P.: The Brauer-Hasse-Noether Theorem in historical perspective., vol. 15 of Schriftenreihe der Heidelberger Akademie der Wissenschaften. Springer, Berlin Heidelberg New York (2005)

24. Schmid, H.L.: Zyklische algebraische Funktionenkörper vom Grade $p^{n}$ über endlichem Konstantenkörper der Charakteristik p. J. reine angew. Math. 175, 108-123 (1936)

25. Segal, S.L.: Mathematicians under the Nazis. Princeton University Press, Princeton, NJ (2003)

26. Serre, J.P.: Corps locaux. Hermann, Paris (1962)

27. Sertöz, A.S.: A Scientific Biography of Cahit Arf (1910-1997). unpublished manuscript (2008)

28. Teichmüller, O.: p-Algebren. Deutsche Math. 1, 362-388 (1936)

29. Teichmüller, O.: Verschränkte Produkte mit Normalringen. Deutsche Math. 1, 92-102 (1936)

30. Witt, E.: Riemann-Rochscher Satz und $\zeta$-Funktion im Hyperkomplexen. Math. Ann. 110, $12-$ 28 (1934)

31. Witt, E.: Theorie der quadratischen Formen in beliebigen Körpern. J. Reine Angew. Math. 176, 31-44 (1937)

32. Witt, E.: Zyklische Körper und Algebren der Charakteristik $p$ vom Grad $p^{n}$. Struktur diskret bewerteter perfekter Körper mit vollkommenem Restklassenkörper der Charakteristik p. J. Reine Angew. Math. 176, 126-140 (1937)

33. Witt, E.: Über eine Invariante quadratischer Formen modulo 2. J. Reine Angew. Math. 193, 119-120 (1954)

34. Kersten, I. (ed.): E. Witt, Collected papers - Gesammelte Abhandlungen. With an essay by Günter Harder on Witt vectors. Springer, Berlin (1998) 